\title{
High rate of popliteal artery injuries and limb loss in 96 knee dislocations
}

\author{
M Held* FC Orth(SA), MMed(Ortho) \\ M Laubscher* FC Orth(SA) \\ $R$ von Bormann* FC Orth(SA) \\ J Walters* FC Orth(SA) \\ S Roche* FC Orth(SA) \\ A Banderker ${ }^{* *}$ FCS(SA), MMed(Surg) \\ P Navsaria** FCS(SA) \\ A Nicol ${ }^{* *}$ FCS(SA), PhD \\ S Maqungo* FC Orth(SA) \\ ${ }^{*}$ Orthopaedic Research Unit \\ **Trauma Centre, Department of Surgery \\ Groote Schuur Hospital, University of Cape Town \\ Corresponding author: \\ Dr Michael Held \\ H49 Old Main Building \\ Groote Schuur Hospital \\ 7925 Observatory \\ South Africa \\ Tel: $0027-214066157$ \\ Fax: $0027-21472709$ \\ Email: michael.held@uct.ac.za
}

\begin{abstract}
Background: Knee dislocations are reported to have an incidence of popliteal artery injuries between $7 \%$ and $64 \%$. The purpose of this study was to determine the incidence of injuries to the popliteal artery as well as early limb loss in knee dislocations at a level-1 trauma hospital in South Africa.

Methods: Twenty-eight patients with popliteal artery injuries were selected from a prospectively collected database of 96 patients with acute knee dislocations. The incidence of vascular injuries, compartment syndromes and limb loss was determined retrospectively.

Results: Of 96 knee dislocations 28 had a popliteal artery injury (29.1\%). Seven of 28 patients with popliteal artery injuries needed an amputation. Of the 28 patients, ten were documented as a threatened limb. Five of these ten patients $(50 \%)$ needed an amputation. The documented median delay to revascularisation of patients who presented with threatened limbs was 10 hours.

Conclusion: Overall, one-third of knee dislocations presented with a vascular injury. The prevalence of amputations in knee dislocations with associated popliteal artery injuries was $25 \%$ and increased to more than $50 \%$ in patients who presented with a threatened limb.
\end{abstract}

Key words: knee dislocation, popliteal artery injury, amputation, limb loss

http:/ / dx.doi.org/10.17159/2309-8309/2016/v15n1a8

\section{Background}

An accurate definition of a knee dislocation is subject to on-going discussions as the description of a knee injury as a dislocation does not clearly define the injury or guide management. ${ }^{1}$ Radiologic evidence of a dislocation is not available in about $20 \%-50 \%$ of the patients, as the knee often reduces spontaneously or is reduced by emergency staff at the scene. Thus, many authors define knee dislocations as the disruption of three or more of the four major knee ligaments. Yet, it has been argued that the disruption of only two ligaments is sufficient for a knee dislocation to occur such as in dislocations with an intact posterior cruciate ligament. ${ }^{2}$ This lack of a commonly accepted definition makes a comparison of incidences of associated injuries challenging and reports of popliteal artery injuries in knee dislocations vary widely between $7 \%$ and $64 \% .^{3-8}$ 
A recent study has described popliteal artery injury rates as low as $1.6 \%$ with only $0.1 \%$ of patients needing an amputation. ${ }^{9}$ Indisputably, each multidirectional knee injury with an adequate history of trauma and presentation should be regarded as a knee dislocation until proven otherwise. ${ }^{1}$

The mechanism of injury in knee dislocations can range from high energy injuries due to road accidents and falls from a height to low energy injuries such as sports injuries and even ultra-low-velocity injuries such as simple falls, especially in obese patients. ${ }^{10-13}$ The energy level of the injury can affect the injury pattern itself, which varies from ligamentous disruption only, to knee dislocations with severe soft tissue damage, compartment syndrome, open wounds and fractures, all of which are risk factors for adverse limb outcomes. ${ }^{14,15}$ In its most severe presentation a knee dislocation can be associated with popliteal artery injuries resulting in a threatened limb and subsequent limb loss if not treated urgently. The specific anatomy of the popliteal artery makes it susceptible to damage during trauma. It is fixed to the distal femur in the adductor canal, and is tethered distally under the soleus muscle and inbetween the two heads of the gastrocnemius muscles. With its tenuous collateral supply, the arterial flow is very rarely sufficient to guarantee adequate distal perfusion to the limb during a complete disruption of the popliteal artery. The purpose of this study was to determine the frequency of popliteal artery injuries in knee dislocations and evaluate the incidence of early limb loss.

\section{Methods}

Twenty-eight of 96 consecutive knee dislocations admitted to the trauma unit of a tertiary care hospital in South Africa over a period of 12 years (2000-2012) were included in this study and were analysed retrospectively. The data was collected prospectively. Included were all patients with knee dislocations diagnosed radiologically or, in patients who presented with a reduced joint, in the presence of multidirectional, gross instability. Children were excluded. The viability of the limb on presentation was documented according to the Rutherford Classification into 'viable', 'threatened' and 'non-viable' limbs. ${ }^{2}$ In accordance with the protocol of the local trauma unit the vascular status was assessed via an angiogram in all patients (Figure 1). A hybrid procedure room for simultaneous computer tomography with angiogram (CTA) in the operating room was not available and therefore, in threatened limbs with vascular hard signs, arterial contrast was injected into the femoral artery and the popliteal artery injury was visualised under fluoroscopy either in the emergency room or on the operating table prior to the intervention ('on-table' angiography). A CT angiogram with venous contrast or digital subtraction angiogram with arterial contrast was obtained in patients, in which a threatened limb was excluded. All vascular injuries were managed by a dedicated trauma team.
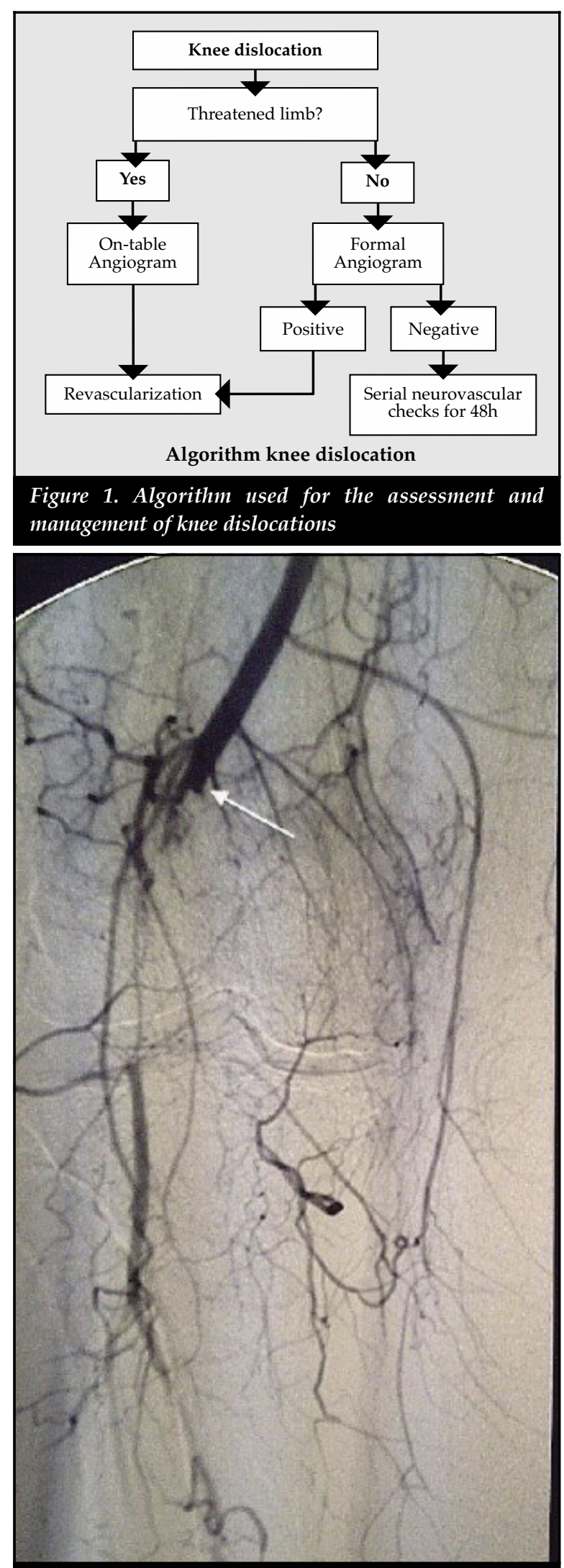

Figure 2. Angiogram; injury to the popliteal artery 
The musculoskeletal pathology was assessed and treated by a specialised orthopaedic unit. All popliteal artery injuries were repaired through a medial approach with end-to-end suturing or grafted with a reverse saphenous vein graft if direct repair was not possible. The knee dislocations were reduced and stabilised with an external fixation device prior the vascular repair. A joint spanning configuration of two $5 \mathrm{~mm}$ half pins each, proximal and distal to the joint, was used. The duration of external fixation was not documented. Capsular structures were not repaired at the initial sitting. A lower leg compartment release was carried out in limbs assessed to have a compartment syndrome. This was a clinical diagnosis with examinations done pre-operatively and at regular 2-hour intervals for 48 hours after surgery and was not confirmed by intra-compartmental pressure measurements. The delay from injury to revascularisation, viability of the limb, type of vascular injury and repair, mechanism of injury, associated compartment syndrome, and limb loss were documented. Ethics approval for the study was obtained.

\section{Results}

In the study period 96 knee dislocations were identified. Twenty-eight $(29.1 \%)$ patients (14 males) had evidence of a popliteal artery injury (Figure 2). This was confirmed by formal digital subtraction angiography in ten cases and by CTA in eight cases. In ten patients an 'on-table' angiogram was performed. In the remaining 68 patients vascular injuries were excluded by angiography. In two cases the diagnosis of a knee dislocation had not been made primarily but only after a popliteal artery injury was detected.

Seven patients of the 28 patients (25\%) with popliteal artery injuries needed an amputation at an average of 9.3 days after the initial assessment (range: 0-42). The mechanisms of injury included 14 falls (50\%), 11 motor vehicle crashes $(39.3 \%)$, and three soccer injuries $(10.7 \%)$. On documentation no distinction was made regarding falls from height or lowenergy falls. Three of the 11 patients (27.3\%) who injured their knee during a motor vehicle crash needed an amputation. Four of the 14 patients $(28.6 \%)$ who dislocated their knee during a fall were amputated; none of the three sport-related injuries needed an amputation (Table I).

An associated popliteal vein injury was found in three patients, two of which needed an amputation $(66.7 \%)$; in the remaining 22 patients $(13.6 \%)$ without an associated popliteal vein injury, three needed an amputation. A compartment syndrome was present in four limbs, of which two were amputated compared to two amputations of the remaining 24 patients $(8.3 \%)$, who did not have a compartment syndrome (Table II).

Of the 28 patients, 18 presented with a 'viable' limb and ten were documented as a threatened limb. Five of the ten patients $(50 \%)$ who presented with a threatened limb needed an amputation (Table II). The documented median delay to revascularisation of patients who presented with threatened limbs was 10 hours (interquartile range
[IQR]: 13.2). Two patients who were revascularised after 12 hours of ischaemic time developed acute kidney injury with myoglobinuria and abnormally high serum potassium levels. One patient was able to undergo delayed amputation at day 10; the other patient succumbed seven days postoperatively due to multi-organ failure. In both cases the knee dislocation was missed as the joint had reduced prior to admission to the emergency unit.

Two cases in the 'viable' limb group of 18 patients needed an amputation on day 16 and day 42 post-operatively. In one patient a late compartment syndrome was missed; the other patient refused relook surgery once a diagnosis of primary graft failure was made.

Restitution of the vascular supply was by direct repair in nine patients $(32.1 \%)$. All limbs in this group were salvaged. Seventeen patients needed a reverse saphenous vein graft $(60.7 \%)$. Of these patients five $(29.4 \%)$ had an amputation (Table III). The popliteal artery was found transected in nine patients $(32.1 \%)$ and thrombosed in six $(21.4 \%)$, and an intimal flap was seen in 11 patients (27.3\%). Percutaneous transarterial repair during arteriography was not performed.

\section{Discussion}

The rate of popliteal artery injuries and amputations in knee dislocations in our patient population remains alarmingly high. Missing knee dislocations and associated injuries poses a high risk of limb loss and can be life-threatening.

\section{Table I: Mechanism of injury}

\begin{tabular}{|l|c|c|}
\hline Mechanism & N (\%) & Limb loss \\
\hline Fall & $14(50 \%)$ & $4 / 14(28.6 \%)$ \\
\hline MVC & $11(39.3 \%)$ & $3 / 11(27.3 \%)$ \\
\hline Soccer & $3(10.7 \%)$ & 0 \\
\hline Total & $\mathbf{2 8}$ & $\mathbf{7 ( 2 5 \% )}$ \\
\hline N = number; MVC = motor vehicle crash \\
\hline
\end{tabular}

\section{Table II: Associated conditions and injuries}

\begin{tabular}{|l|c|c|}
\hline Associated conditions & $\mathbf{N}(\%)$ & Limb loss \\
\hline Popliteal vein & $3 / 28(10.7 \%)$ & $2 / 3(66.7 \%)$ \\
\hline Compartment syndrome & $4 / 28(14.3 \%)$ & $2 / 4(50 \%)$ \\
\hline Threatened limb & $10 / 28(35.7 \%)$ & $5 / 10(50 \%)$ \\
\hline
\end{tabular}

\section{Table III. Vascular repair}

\begin{tabular}{|l|c|c|}
\hline Type of repair & $\mathbf{N}(\%)$ & Limb loss \\
\hline Direct & $9 / 28(32.1 \%)$ & 0 \\
\hline RSVG & $17 / 28(60.7 \%)$ & $5 / 17(29.4 \%)$ \\
\hline RSVG = reverse saphenous vein graft \\
\hline
\end{tabular}


Therefore, the most severe form of knee dislocation should be assumed until further examination and special investigations have ruled out associated injuries.

In two patients of our study a knee dislocation was missed as the joint had reduced prior to admission to the emergency unit and a multidirectional instability of the knee was overlooked. This might point to a much larger number of knee dislocations without vascular injuries, which are missed on admission. In our study 28 of 96 knee dislocations were found to have a popliteal artery injury $(29.1 \%)$. This incidence is comparable to above-mentioned studies although a Finnish population-based study has reported popliteal artery injury rates as low as $1.6 \%$ with only $0.1 \%$ of limb loss. ${ }^{9}$ Forty-six per cent of knee dislocations were caused by falls on the same level and the lower rate of popliteal artery injury might be a consequence of this predominantly low-energy injury pattern. Our study was done in a cohort recruited from a single centre and subspecialised Level 1 Trauma Unit, rather than being a population-based study and with this we might have selected more severe injury patterns. Yet, we also acknowledge our numbers could be lower if missed knee dislocations were to be taken into account.

It remains controversial whether distal perfusion should be assessed by means of physical examination (with or without the measurement of Ankle-Brachial Index), or routine arteriography. ${ }^{16}$ Most cases with threatened limbs present with vascular hard signs or symptoms described by de Bakey $^{17}$ such as active haemorrhage, expanding haematoma, bruit or thrill, and distal ischaemia with symptoms like absent pulses, pain, paraesthesia, paralysis and paleness. But often only subtle signs such as a diminished pulse amplitude and pain can be found, so that a high index of suspicion is needed to avoid missing vascular damage.

Many authors agree that patients with a viable limb and patent distal pulses should be reviewed regularly for 48 hours and treated conservatively unless deterioration is evident. ${ }^{18}$ With adequately trained staff and appropriate facilities, acceptable results have been reported with serial neurovascular examinations including Ankle-Brachial Index (ABI) measurements for at least 24 hours. ${ }^{18}$ This seems advisable since the presence of normal pulses on admission does not rule out a clinically significant vascular injury. ${ }^{16,19,20}$ Cases in which a viable limb shows an ABI of less than 0.90 warrant further vascular imaging and reperfusion. Other options such as duplex ultrasonography $(100 \%$ sensitivity and $97 \%$ specificity for clinically significant arterial injury) or CT angiography (95-100\% sensitivity and $97-98 \%$ specificity) have also been described. ${ }^{21-23}$

Even though, serial neurovascular examinations have shown equal sensitivity and specificity compared to obtaining a formal angiographic study, we have adopted a policy that ensures angiographic examination of all knee dislocations. Despite these efforts our amputation rate remains at $25 \%$ (seven of 28 patients) of diagnosed vascular injuries.
A conservative approach to non-occlusive popliteal artery injuries with serial neurovascular examinations have been proposed by some centres with an adequate set-up. This seems to be more cost effective and has less morbidity for the patient than surgical exploration and repair of the artery with a similar outcome. ${ }^{24}$ Contrary to this, all of the patients who presented with a vascular injury to our unit, occlusive or non-occlusive, underwent vascular repair. Although no clear evidence is available yet, the authors feel that serial examinations are often of insufficient quality, and the costs and morbidity of an early exploration of the popliteal artery outweighs the risk taken if the patient deteriorates unnoticed.

One of the most influential factors for limb salvage is the delay from injury to reperfusion. It is reported that if reperfusion is achieved within 6 hours, amputation rates can be kept below $10 \%$. The amputation rate increases to more than $80 \%$ if the ischaemic time reaches 8 hours. ${ }^{24}$ In our series, five of ten threatened limbs could be salvaged even after an ischaemic time of 10.8 hours (median, IQR: 9.2), but with five patients the sample size was too small to make conclusions on time to revascularisation without bias. A delay to revascularisation will most likely lead to a high risk of reperfusion injury and subsequent multi-organ dysfunction. The exact point of transition from a threatened pulseless limb with strong vascular hard signs to a non-viable limb cannot be measured clinically and it is therefore extremely challenging for the surgeon and the patient to agree on the indication for amputation. Factors such as overall trauma load, associated injuries to bone and soft tissue as well as age of the patient and comorbidities add to the complexity of this decision. Two of our patients who were revascularised after 10 hours developed acute kidney injury. One of these patients died seven days post-operatively due to multi-organ failure, highlighting the danger of this endeavour.

A shortcoming of our study is that we might have missed more knee dislocations and therefore are overreporting the incidence of popliteal artery injuries and amputations. Although we report on one of the highest number of knee dislocations for a single centre, the small number of amputations makes it impossible to reach strong conclusions on associated injuries and reasons for amputations. We also note that we have not differentiated falls into high-energy, low-energy and even ultralow energy falls and cannot assess differences here.

\section{Conclusion}

The incidence of vascular injuries in knee dislocations reached nearly $30 \%$. The risk of amputations in knee dislocations with associated popliteal artery injuries was 25\% and increased to more than $50 \%$ in patients who presented with a threatened limb. 


\section{Conflict of interest statement}

All authors declare that they do not have conflict of interest. The study was approved by the appropriate ethics committee and has therefore been performed in accordance with the ethical standards laid down in the 1964 Declaration of Helsinki and its later amendments.

\section{References}

1. Liow R, McNicholas M, Keating J, Nutton R. Ligament repair and reconstruction in traumatic dislocation of the knee. Journal of Bone $\mathcal{E}$ Joint Surgery, British Volume. 2003;85(6):845-51.

2. Cooper DE, Speer KP, Wickiewicz TL, Warren RF. Complete knee dislocation without posterior cruciate ligament disruption: A report of four cases and review of the literature. Clinical orthopaedics and related research. 1992;284:228-33.

3. Boisrenoult $\mathrm{P}$, Lustig S, Bonneviale P, Leray E, Versier G, Neyret $P$, et al. Vascular lesions associated with bicruciate and knee dislocation ligamentous injury. Orthopaedics $\mathcal{E}$ Traumatology: Surgery \& Research. 2009;95(8):621-26.

4. Engebretsen L, Risberg MA, Robertson B, Ludvigsen TC, Johansen S. Outcome after knee dislocations: a 2-9 years follow-up of 85 consecutive patients. Knee Surgery, Sports Traumatology, Arthroscopy. 2009;17(9):1013-26.

5. Eranki V, Begg C, Wallace B. Outcomes of operatively treated acute knee dislocations. The Open Orthopaedics Journal. 2010;4:22.

6. Nicandri GT, Chamberlain AM, Wahl CJ. Practical management of knee dislocations: a selective angiography protocol to detect limb-threatening vascular injuries. Clinical Journal of Sport Medicine. 2009;19(2):125-29.

7. Patterson BM, Agel J, Swiontkowski MF, MacKenzie EJ, Bosse MJ, Group LS. Knee dislocations with vascular injury: outcomes in the Lower Extremity Assessment Project (LEAP) Study. Journal of Trauma and Acute Care Surgery. 2007;63(4):855-58.

8. Plancher KD, Siliski J. Long-term functional results and complications in patients with knee dislocations. The journal of knee surgery. 2008;21(4):261-68.

9. Sillanpää PJ, Kannus P, Niemi ST, Rolf C, Felländer-Tsai L, Mattila VM. Incidence of knee dislocation and concomitant vascular injury requiring surgery: a nationwide study. Journal of Trauma and Acute Care Surgery. 2014;76(3):715-19.

10. Azar FM, Brandt JC, Miller RH, Phillips BB. Ultra-lowvelocity knee dislocations. The American Journal of Sports Medicine. 2011;39(10):2170-74.

11. Georgiadis AG, Mohammad FH, Mizerik KT, Nypaver TJ, Shepard AD. Changing presentation of knee dislocation and vascular injury from high-energy trauma to lowenergy falls in the morbidly obese. Journal of Vascular Surgery. 2013;57(5):1196-203.

12. Marin EL, Bifulco SS, Fast A. Obesity: A risk factor for knee dislocation. American Journal of Physical Medicine $\mathcal{E}$ Rehabilitation. 1990;69(3):132-34.
13. Peltola EK, Lindahl J, Hietaranta H, Koskinen SK. Knee dislocation in overweight patients. American Journal of Roentgenology. 2009;192(1):101-106.

14. Farber A, Tan TW, Hamburg NM, Kalish JA, Joglar F, Onigman $\mathrm{T}$, et al. Early fasciotomy in patients with extremity vascular injury is associated with decreased risk of adverse limb outcomes: a review of the National Trauma Data Bank. Injury. 2012;43(9):1486-91. Epub 2011/07/02. doi: 10.1016/j.injury.2011.06.006. PubMed PMID: 21719009; PubMed Central PMCID: PMCPmc3777619.

15. Hafez HM, Woolgar J, Robbs JV. Lower extremity arterial injury: Results of 550 cases and review of risk factors associated with limb loss. Journal of Vascular Surgery. 33(6):1212-19. doi: 10.1067/ mva.2001.113982.

16. Stannard JP, Sheils TM, Lopez-Ben RR, McGwin Jr G, Robinson JT, Volgas DA. Vascular injuries in knee dislocations: The role of physical examination in determining the need for arteriography. The Journal of Bone \& Joint Surgery. 2004;86(5):910-15.

17. DeBakey ME, Simeone FA. Battle injuries of the arteries in World War II: An analysis of 2,471 cases. Annals of Surgery. 1946;123(4):534.

18. Hollis JD, Daley BJ. 10-year review of knee dislocations: is arteriography always necessary? Journal of Trauma and Acute Care Surgery. 2005;59(3):672-76.

19. Rose SC, Moore EE. Trauma angiography: the use of clinical findings to improve patient selection and case preparation. Journal of Trauma and Acute Care Surgery. 1988;28(2):240-45.

20. Snyder WH, Thal ER, Bridges RA, Gerlock AJ, Perry MO, Fry WJ. The validity of normal arteriography in penetrating trauma. Archives of Surgery. 1978;113(4):42428.

21. Fry WR, Smith RS, Sayers DV, Henderson VJ, Morabito DJ, Tsoi EK, et al. The success of duplex ultrasonographic scanning in diagnosis of extremity vascular proximity trauma. Archives of Surgery. 1993;128(12):1368-72.

22. Inaba K, Potzman J, Munera F, McKenney M, Munoz R, Rivas L, et al. Multi-slice CT angiography for arterial evaluation in the injured lower extremity. Journal of Trauma and Acute Care Surgery. 2006;60(3):502-507.

23. Soto JA, Múnera F, Cardoso N, Guarín O, Medina S. Diagnostic performance of helical $\mathrm{CT}$ angiography in trauma to large arteries of the extremities. Journal of Computer Assisted Tomography. 1999;23(2):188-96.

24. Green NE, Allen BL. Vascular injuries associated with dislocation of the knee. The Journal of Bone \& Joint Surgery. 1977;59(2):236-39.

This article is also available online on the SAOA website (www.saoa.org.za) and the SciELO website (www.scielo.org.za). Follow the directions on the Contents page of this journal to access it. 